\title{
Speedier discharges: a simple project to identify ways to expedite inpatient discharges
}

\author{
Authors: Sushuma Kalidindi, ${ }^{*}$ Christina Longhurst and Zia Din
}

\section{Introduction}

Delays in inpatients' discharges can have a major effect on patient flow and patient experience, as well as a financial burden on the trust. ${ }^{1}$ The aims of our project were to:

$>$ discover if there were any delays to patient discharges

$>$ detect any themes good or bad which enabled or hindered the discharge process.

\section{Methods}

After discussion with all members of the multidisciplinary teams and discharge facilitators, a questionnaire was developed. An audit of all patients discharged from the medical division over 24 hours was undertaken using the questionnaire and medical notes. Further information was obtained from our in-house information technology system. An MS Excel spreadsheet and BaseLine ${ }^{\odot}$ were used for data analysis.

\section{Results}

A total of 34 patients were identified. The following themes were noted:

> Decision to discharge to actual discharge: There was a wide variation in time from decision to discharge until patient leaving hospital (30 minutes to 9 hours). We had confirmed our preexisting suspicion of delays in obtaining hospital transport as a main cause of delay. Other factors identified included delay in writing discharge letters.

> Pharmacy: Only four patients had their 'to take outs' (TTOs) dispensed before the day of discharge and a further three patients didn't need medicines to take home. The majority of TTOs were dispensed from our dedicated mini pharmacy hubs with an average turnaround time of 15 mins and a maximum of 3 hours 25 minutes. To avoid delays in dispensing, our trust has developed an innovative dedicated mini pharmacy hub on each floor which expedites issuance of TTOs compared with the main pharmacy.

> Discharge lounge: Initially only two patients out of 34 used our discharge lounge (both from the same ward), according to the medical notes. However, on review of the discharge lounge records a further six were sent there. This identified the need for better documentation, which has been a consistent thread throughout. One ward reported not using the discharge lounge as they were not picking up the telephone.

\section{Conclusion}

A thorough review of our current discharges yielded a wide variation in different practices on different wards. To spread best practice, we recommend a focus group of all discharge facilitators to spread awareness of good practice to other wards. It also highlighted delays in completing discharge letters due to staffing levels and current work pressures on junior doctors. We are currently considering alternatives, including training admin professionals/ discharge facilitators to complete discharge letters. ${ }^{2}$ We have introduced the use of mini pharmacy hubs in each area of the hospital which allow medications to be dispensed much more quickly. It also highlighted transport as a major issue in delay in discharging patients. This has now been corrected by optimising our transport team.

This project highlights the importance of reviewing current process to see if they can be optimised. We are planning to re-audit in a few months to see if discharges can be further expedited as earlier discharge of just 1-2 patients per ward by a few hours can have a major financial impact along with patient flow and improvement in patient experience.

\section{References}

1 Powell ES. The relationship between inpatient discharge timing and emergency department boarding. J Emerg Med 2012;42:186-96.

2 Knowledge HSJ. How a new administrative role is helping improve patient care. Online. HSJ, 2016. www.hsj.co.uk/hsj-knowledge/howa-new-administrative-role-is-helping-improve-patient-care/7001150. article

Authors: University Hospital North Midlands

${ }^{*} \mathrm{RCP}$ chief registrar 